\title{
Exploring the Global Topology of the Universe
}

\author{
Helio V. Fagundes \\ Instituto de Física Teórica, Universidade Estadual Paulista \\ São Paulo, SP 01405-900, Brazil
}

Received on 20 December, 2001

\begin{abstract}
We review the work done by our group on cosmic topology. It ranges from early attempts to solve a famous controversy about quasars through the multiplicity of images, to quantum cosmology in this context and an application to QED renormalization.
\end{abstract}

\section{Introduction}

The preferred cosmological models for the description of the universe, except for its very first instants, are those of Friedmann-Lemaitre-RobertsonWalker (FLRW), popularly known as 'Big Bang' models. See, for example, Ohanian and Ruffini [1].

It is generally assumed that the global topology of cosmic spatial sections is simply connected, that is, they are one of the spherical $\left(S^{3}\right)$, Euclidean $\left(E^{3}\right)$, or hyperbolic $\left(H^{3}\right)$ spaces, which have positive, null, and negative curvature, respectively.

But spaces $E^{3}$ and $H^{3}$ have infinite extension. This has led some pioneers, like Einstein, Schwarzschild, and Friedmann, to consider the question of the finite vs. infinite size of the universe, and its topology.

Beginning in 1971, with a paper by Ellis [2], research on the possibility of cosmic space being finite and its topology multiply connected, has been done more systematically, if slowly at first.

Today there is a reasonable number of researchers in this area (my guess is about a hundred worldwide), including here in Brazil.

Since this area is still little known, in this talk I will present a summary of work done by myself and a few collaborators at IFT/UNESP in São Paulo. The sections that follow list a number of published papers, which are representative of the effect of nontrivial cosmic topology on the topics: section II, formal theoretical works; III, the quasar redshift controversy; IV, fitting models to data; V, cosmic crystallography; VI, quantum field theory and quantum cosmology; and VII, the cosmic microwave background.

Note that most of this research was done before 1998 , so we could with impunity assume a null cosmological constant.

\section{Formal theoretical works}

II.1 A cosmological model with compact space sections and low mass density[3].

In this model the adopted spacetime topology was $M^{4}=R \times \Sigma$, where $R$ is the time axis and $\Sigma$ is the space section. This form of spacetime holds for all works cited below (with different $\Sigma$ 's, of course).

Here $\Sigma=T_{g} \times S^{1}$, where $T_{g}$ is the genus- $g$ surface and $S^{1}$ is the circle. The metric is

$$
d s^{2}=a^{2}(\eta)\left(d \eta^{2}-d \rho^{2}-\sinh ^{2} \rho d \varphi^{2}\right)-b^{2}(\eta) d \zeta^{2},
$$

where

$$
\begin{aligned}
c t(\eta) & =a_{*}(\sinh \eta-\eta), \\
a(\eta) & =a_{*}(\cosh \eta-1), \\
b(\eta) & =3 a_{*}[\eta \operatorname{coth}(\eta / 2)-2],
\end{aligned}
$$

with $a_{*}=$ constant. This metric had been studied by Kantowski and Sachs [4], and is of type III in the classification of Bianchi [5].

At the time I was unaware of the existence of threedimensional closed hyperbolic manifolds, and this was the best substitute I could find for a closed Friedmann model of undercritical density.

\section{II.2 Compactification of Friedmann's hyperbolic model[6]}

Now $\Sigma$ is a closed hyperbolic manifold constructed by Best [7]. I did not go into the details of image formation, but found that the space of images was the covering space $H^{3}$, and that homogeneity and isotropy should be reinterpreted in terms of the distribution of multiple images in the covering space. I also found that we should have the relative density of matter $\Omega_{0}<0.964$ in order that a repeated pattern of images could be observed.

II.3 Relativisic cosmologies with closed, locally homogeneous spatial sections [8] 
Here I established a correspondence between the geometric classifications of Bianchi and Kantowski-Sachs (BKS) on one side, and Thurston's on the other, according to the following table:

\begin{tabular}{|c|c|}
\hline \hline Thurston type & BKS type \\
\hline T1 & BIX \\
T2 & BI, BVII $(0)$ \\
T3 & BV, BVII $(\mathrm{A}>0)$ \\
T4 & KS \\
T5 & BVI $(1)$ \\
T6 & BVIII \\
T7 & BII \\
T8 & BVI $(0)$ \\
\hline \hline
\end{tabular}

It was also found that Bianchi types $I V$ and $V I(A), 0<A<1$, cannot be compactified - i. e., there are not any closed, locally homogeneous manifolds of these Bianchi types.

\section{II.4 Closed spaces in cosmology $[9]$}

This paper improves on the previous one, and discusses the question of local vs. global homogeneity of the constant curvature models.

\section{II.5 Numerical study of a perturbed Einstein-de Sitter cosmological model[10]}

Here $\Sigma$ is the 3 -torus $T^{3}$. Our purpose was to find how an inhomogeneity of the matter density, written as $\rho(x, \tau), \tau=1-t$, affects the metric, which was assumed to have the form

$$
d s^{2}=c^{2} d \tau^{2}-a^{2}(x, \tau) d x^{2}-b^{2}(x, \tau)\left(d y^{2}+d z^{2}\right),
$$

where $a^{2}(x, \tau), b^{2}(x, \tau)$ reduce to $a_{E d S}(t)=\left(t / t_{0}\right)^{2 / 3}$ if we remove the perturbation. The result was that, for a perturbation of $\rho$ of about $80 \%$, the metric fluctuated at most $5 \%$, in agreement with a heuristic estimate of Barrow [11]

\section{The quasar redshift contro- versy}

III.1 Quasar-galaxy associations with discordant redshifts as a topological effect. II. A closed hyperbolic model[12]

$\Sigma$ is one of Best's [7] manifolds with a regular icosahedron as fundamental polyhedron (FP). The metric is the same as in Friedmann's open model.

The search for images in conjunction to simulate the controversial pairs was done by computer, by scanning all directions that met the observer. The obtained pairs were not realistic: e. g., redshifts 0.12 for the galaxy and 4.3 for the quasar.
III.2 Smallest universe of negative curvature[13]

$\Sigma$ is the smallest known CHM, with normalized volume $0.9427 \ldots$ and 18 faces, discovered independently by Weeks [14] and by Matveev and Fomenko [15]. It was assumed $\Omega_{0}=0.1$. Here I got better results for the quasar-galaxy associations than in the previous case: many of the simulated pairs had realistic redshifts, like 0.002 for the galaxy and 1.31 for the quasar or quasistellar object (QSO).

However, comparison with Burbidge et al.'s [16] catalog of associations, for example, would still not be reliable: the model parameters are arbitrary, only 31 sources were used in the simulation, and of course many conjunctions are line-of-sight concidences.

The model is also interesting on account of the small volume: multiple images would allow astronomers to see objects at different epochs of their evolution; and it has a larger probability than a larger model for the spontaneous creation of the universe.

\section{Fitting models to data}

\section{IV.1 A search for QSO's to fit a cosmological model with flat, closed spatial sections[17]}

Based on a catalog with about 1500 quasars, we looked for pairs equidistant from Earth, and along three orthogonal axis. $\Sigma$ was the 3 -torus, with a rectangular parallepiped as FP, whose sides were fitted to 3591 , 2966, and $2792 \mathrm{Mpc}$.

\section{IV.2 A suggestion on the pair of QSO triplets $1130+106\{B, A, C\},\{X, Y, Z\}[18]$}

The quasars in the title are two aligned triplets discovered by Arp and Hazard [19], with similar corresponding redshifts, which are $\{2.1,0.54,1.6\}$ for the first triplet, and $\{2.1,0.51,1.7\}$ for the second one. They were adjusted to an Einstein-de Sitter model with a cube of side $387 \mathrm{Mpc}$ as FP, which is an unrealistically small size.

\section{Cosmic crystallography}

\section{V.1 On closed Einstein-de Sitter universes[20]}

The method of cosmic crystallography (CC) introduced by Lachièze-Rey et al. [21] was applied to a few models, all with Einstein-de Sitter metric but varying topologies. The idea was to verify that the $\mathrm{CC}$ method yields observable (in principle) results, even if the FP's sides are of the order of the horizon's radius $R_{H}=2 c / H_{0}$, where $H_{0}$ is Hubble's constant. Good results were obtained with the FP's sides equal $0.7 H_{0}$, and not so good ones with sides equal $1.2 H_{0}$ 
V.2 Cosmic crystallography in a compact hyperbolic universe [22]

$\Sigma$ and FP are the same as in [12]. We applied the CC method to this geometrically inhomogeneous model and did not obtain any sharp peaks - as expected from the independent works of Lehoucq et al. [23] and Gomero et al. [24].

But we did get a smaller fluctuation that is not present in the control open cosmology. We are at present tentatively attributing these small fluctuations to type II pairs, as defined in [23].

\section{Quantum field theory and quantum cosmology}

VI.1 A cosmic lattice as the substratum of quantum fields[25]

This work is a lowest-order attempt to avoid the infinities of quantum electrodynamics renormalization. Both configuration and momentum spaces are assumed to have the topology of a 3-torus $T^{3}$, with cubes of sides $a=c / H_{0}=8000 \mathrm{Mpc}=2.7 \times 10^{28} \mathrm{~cm}$, and $P=2 \pi \hbar / a$ $\approx 10^{20} \mathrm{Gev} / c$, respectively.

Lorentz invariance is abandoned for very large, presently inaccessible energies.

For charge and mass renormalization at the oneloop order, I got $Z_{3}=0.925, e=\sqrt{Z_{3}} e_{0}=0.962 e_{0}$, $m=1.185 m_{0}$, and $Z_{2}=1.160$, in the notation of Itzykson and Zuber [26].

VI.2 Casimir energy in a small volume, multiply connected, static hyperbolic preinflationary universe[27]

This work was orally communicated at this Meeting, and appears elsewhere in these Proceedings.

VI.3 Birth of a closed universe of negative spatial curvature [28]; On the birth of a closed hyperbolic universe[29]

$\Sigma$ is the first the lens space $L(50,1)$, then Weeks manifold as in [13].

Starting with the spontaneous creation, from a spherical orbifold, of a de Sitter universe with the topology of a lens space, we proceeded as De Lorenci et al. [30], to obtain a quantum topology change into a de Sitter universe with Weeks manifold as space section. After inflation it becomes a closed hyperbolic universe, with the metric of an open FLRW universe.

\section{The cosmic microwave back- ground radiation}

VII.1 The quadrupole component of the relic radiation in a quasi-hyperbolic cosmological

\section{$\operatorname{model}[31]$}

This was an application of the model in [3] to a quadrupole moment of the cosmic microwave background reported by Fabbri and Melchiorri [32]. Comparing the obtained relation

$$
1+Z=\frac{a\left(\eta_{\text {observ }}\right)}{a\left(\eta_{\text {emission }}\right)}\left(1-\varepsilon \cos ^{2} \theta_{\text {observ }}\right)
$$

with the result of [32], I obtained $\varepsilon=4 Q / 2.7 \mathrm{~K}=$ $0.0013, \Omega_{0}=1-3 \varepsilon / 2=0.998$.

VII.2 Fitting hyperbolic universes to CayónSmoot spots in COBE maps[33]; Sources of CMB spots in closed hyperbolic universes [34]

Cayón and Smoot [35] identified several spots in NASA's satellite COBE's maps of the cosmic microwave background as physical (rather than noise), hence as small fluctuations of the matter density.

In these papers I simulated the position of six cold and eight hot CS spots in closed hyperbolic manifolds, and interpreted them as having evolved into today's galaxy superclusters and galaxy voids, respectively.

Present catalogs [36] only list superclusters up to $Z=0.12$, which is completely inadequate for a fit of the simulated models: typically, in one of the latter the 14 redshifts are in the range 0.361 to 1.370 .

I thank the Brazilian agency $\mathrm{CNPq}_{\mathrm{q}}$ for partial financial support.

\section{References}

[1] H. Ohanian and R. Ruffini, Gravitation and Spacetime (W. W. Norton, New York, 1994), Chapter 9.

[2] G. F. R. Ellis, Gen. Relat. Grav. 2, 7 (1971).

[3] H. V. Fagundes, Lett. Math. Phys. 6, 417 (1982).

[4] R. Kantowski and R. K. Sachs, J. Math. Phys. 7, 443 (1966).

[5] L. Bianchi, Mem. Mat. Fis. Soc. Ital. Sci. 11, 267 (1898).

[6] H. V. Fagundes, Phys. Rev. Lett. 51, 517 (1983).

[7] L. A. Best, Can. J. Math. 23, 451 (1971).

[8] H. V. Fagundes, Phys. Rev. Lett. 54, 1200 (1985).

[9] H. V. Fagundes, Gen. Relat. Grav. 24, 199 (1992); Addendum in Gen. Relat. Grav. 30, 1437 (1998).

[10] H. V. Fagundes and S. F. Kwok, Astrophys. J. 368, 337 (1991)

[11] J. D. Barrow, Quart. J. Roy. Astr. Soc. 30, 163 (1989).

[12] H. V. Fagundes, Astrophys. J. 338, 615 (1989); Errata in Astrophys. J. 349, 678 (1990).

[13] H. V. Fagundes, Phys. Rev. Lett. 70, 1579 (1993). 
[14] J. R. Weeks, Ph. D. Thesis, Princeton University (1985); SnapPea: A computer program for creating and studying three-manifols, freely available from the site www . northnet.org/weeks.

[15] S. V. Matveev and A. T. Fomenko, Russian Math. Surveys 43, 3 (1988).

[16] G. Burbidge, A. Hewitt, J. V. Narlikar, and P. Das Gupta, Astrophys. J. (Suppl.) 74, 675 (1990).

[17] H. V. Fagundes and U. F. Wichoski, Astrophy. J. 322, L5 (1987)

[18] H. V. Fagundes, Rev. Mex. Astron. Astrof. 21, 79 (1990).

[19] H. Arp and C. Hazard, Astrophys. J. 240, 726 (1980).

[20] H. V. Fagundes and E. Gausmann, Phys. Lett. A238, 235 (1998).

[21] M. Lachièze-Rey, J.-P. Luminet, and J.-P. Uzan, Astron. Astrophys. 313, 339 (1996).

[22] H. V. Fagundes and E. Gausmann, Proc. XIX Texas Symp. Relat. Astrophys. Cosmol. (Paris, 1998), Nucl. Phys. B (Proc. Suppl.) 80, CD-ROM.

[23] R. Lehoucq, J.-P. Luminet, and J.-P. Uzan, Astron. Astroph. 344, 735 (1999).

[24] G. Gomero, A. F. Teixeira, M. Rebouças, and A. Bernui, preprint gr-qc/9811038 (1998).

[25] H. Fagundes, Rev. Bras. Fís. (now Braz. J. Phys.) 19, $27(1989)$
[26] C. Itzykson and J.-B. Zuber, Quantum Field Theory (Addison-Wesley, New York, 1980), Chapter 7.

[27] D. Müller, H. V. Fagundes, and R. Opher, Phys. Rev. D63, 123508 (2001).

[28] S. S. e Costa and H. V. Fagundes, Gen. Relat. Grav. 30, 863 (1999).

[29] S. S. e Costa and H. V. Fagundes, Gen. Relat. Grav. 33, 1489 (2001).

[30] V. A. De Lorenci, J. Martin, N. Pinto-Neto, and I. D. Soares, Phys. Rev. D56, 3329 (1997).

[31] H. V. Fagundes, Astrophys. Lett. (now Astrophys. Lett. Comm.) 23, 161 (1983).

[32] R. Fabbri and F. Melchiorri, Gen. Relat. Grav. 13, 201 (1981).

[33] H. V. Fagundes, Astrophys. J. 470, 494 (1996).

[34] H. V. Fagundes, preprint astro-ph/0007443; to appear in Proc. IX Marcel Grossmann Meeting on General Relativity, eds. R. T. Jantzen, V. Gurzadyan, and R. Ruffini (World Scientific, Singapore, 2002).

[35] L. Cayón and G. Smoot, Astrophys. J. 452, 494 (1996).

[36] M. Einasto, E. Tago, J. Jaaniste, J. Einasto, and H. Andernach, Astron. Astrophys. 123, 119 (1997). 